\title{
The mGluR5 antagonist AFQ056 does not affect methylation and transcription of the mutant FMR1 gene in vitro
}

\author{
Elisabetta Tabolacci', Filomena Pirozzi ${ }^{1}$, Baltazar Gomez-Mancilla ${ }^{2}$, Fabrizio Gasparini ${ }^{2}$ and Giovanni Neri ${ }^{1,3^{*}}$
}

\begin{abstract}
Background: Fragile $X$ syndrome (FXS), the leading cause of inherited mental retardation, is due to expansion and methylation of a CGG sequence in the FMR1 gene, which result in its silencing and consequent absence of FMRP protein. This absence causes loss of repression of metabotropic glutamate receptor 5 (mGluR5)-mediated pathways resulting in the behavioral and cognitive impairments associated with FXS. In a randomized, double-blind trial it was recently demonstrated a beneficial effect of AFQ056, a selective inhibitor of metabotrobic glutamate receptor type 5 (mGluR5), on fully methylated FXS patients respect to partially methylated FXS ones.

Methods: To determine whether AFQ056 may have secondary effects on the methylation and transcription of FMR1, here we treated three FXS lymphoblastoid cell lines and one normal control male line. A quantitative RT-PCR was performed to assess transcriptional reactivation of the FMR1 gene. To assess the methylation status of the FMR1 gene promoter it was carried out a bisulphite sequencing analysis.

Results: Both FMR1-mRNA levels and DNA methylation were unmodified with respect to untreated controls.

Conclusions: These results demonstrate that the AFQ056 effect on fully methylated FXS patients is not due to a secondary effect on DNA methylation and consequent transcriptional activation of FMR1.
\end{abstract}

Keywords: Fragile X syndrome, AFQ056, mGluR5 inhibitors, DNA methylation, Epigenetic modification

\section{Background}

The fragile X syndrome (FXS, MIM \#300624), the most common cause of inherited mental retardation, is due to the amplification (> 200 repeats) of a sequence of CGG triplets in the 5' UTR of the FMR1 gene, followed by methylation of cytosines, including those of the promoter upstream [1]. Although the coding region of the gene remains intact, the two changes, one structural and one epigenetic, lead to transcriptional silencing, and consequent absence of the FMRP protein, responsible for the manifestations of the syndrome. FMRP is an RNA-binding protein, which inhibits the translation of messenger RNAs (mRNAs), especially within postsynaptic vesicles of the dendritic spines of hippocampal neurons [2]. It has been demonstrated that the absence of FMRP causes an upregulation of metabotropic

\footnotetext{
* Correspondence: gneri@rm.unicatt.it

${ }^{1}$ Istituto di Genetica Medica, Università Cattolica del S. Cuore, Rome, Italy Full list of author information is available at the end of the article
}

glutamate receptors 5 (mGluR5)-mediated signalling pathways, which is the probable cause of the behavioural and cognitive impairments observed in FXS patients [3]. In FXS animal models it was demonstrated that many aspects of the phenotype (behavioural abnormalities, learning deficit, altered dendritic spines, macroorchidism) may be due to excessive mGluR5 signalling. Indeed, crossing Fmr1 KO mice with heterozygous Grm5 KO mice, expressing reduced amounts of glutamate receptors, rescues many of the FXS phenotypes, except for macroorchidism [4]. Consequently, the use of mGluR5 antagonists may represent an effective treatment for many FXS symptoms. The use of 2-methyl-6-(phenylethynyl)-pyridine (MPEP), a prototypic mGluR5 inhibitor, rescues hyperactivity and audiogenic seizures in Fmr1 KO [5]. Recently, in a study performed on Fmr1 $\mathrm{KO}$ mice, AFQ056, a subtype-selective inhibitor of mGluR5, rescued the inhibition of the startle response after a prepulse, while cultured hippocampal neurons

\section{Ciomed Central}


showed shortened dendritic spines [6]. A clinical trial was recently performed to assess the safety and tolerability of AFQ056 in FXS patients, as well as its possible beneficial effect on the behavioural phenotype [7]. This randomized, double-blind, placebo-controlled, crossover study was performed on 30 FXS male subjects. Seven of these, who were carriers of a fully methylated FMR1 mutation, with no detectable production of FMR1-mRNA, showed a significant improvement of their behaviour, as measured with the $\mathrm{ABC}-\mathrm{C}$ scale, after treatment with AFQ056, compared to the placebotreated controls. No response was detected in FXS subjects who carried a partially methylated FMR1 full mutation. This unanticipated finding begs the question whether AFQ056 may have an indirect or secondary effect on methylation of the mutant FMR1 gene and, consequently, on its transcription.

To answer this question we studied the effect of AFQ056 on FMR1 promoter methylation and mRNA production in three FXS lymphoblastoid cell lines with different degrees of DNA methylation (two fully and one partially methylated) and in one normal control line. No demethylation was induced by the treatment with AFQ056 and the levels of FMR1-mRNA remained unmodified. These findings support the conclusion that the AFQ056 effect observed in fully methylated patients is not due to a change in the methylation on the FMR1 gene, but may result from the interaction of AFQ056 with other, yet unknown, target proteins.

\section{Methods}

Lymphoblastoid cell lines were established by EpsteinBarr virus (EBV) transformation from peripheral blood lymphocytes of a normal control male (WT) and three FXS males, with CGG expansion of 250 (E3), 450 (S1) and 100-960 (MP, premutation/full mutation mosaic) repeats, respectively. Lymphoblasts were grown in RPMI1640 medium supplemented with 10\% fetal bovine serum, $2.5 \%$ of L-glutamine and penicillin/streptomycin at $37^{\circ} \mathrm{C}$ with $5 \% \mathrm{CO}_{2}$. Treatments were done in T75 flask containing approximately $20 \times 10^{6}$ cells in a volume of $20 \mathrm{ml}$. The effect of a single treatment with AFQ056 (Novartis) was assessed at various times and concentrations. The drug was added daily at either $1,10,100$ or $1,000 \mu \mathrm{M}$ concentration and cells were harvested to extract RNA and DNA after 3 or 8 days from the beginning of the treatment. Control cultures were sham-treated with the drug diluent. As positive controls for the FMR1 reactivation, parallel cultures were also treated with $1 \mu$ M 5-aza-2-doxycytydine (5-azadC) (SigmaAldrich). Cell viability was assessed after 8 days of treatment using NucleoCounter ${ }^{\circledR}$ (Chemometec, Denmark).

Total RNA from treated and untreated cell lines was extracted with the single-step acid phenol method using
Trizol (Invitrogen), reverse-transcribed to cDNA by MoMLV-RT (Invitrogen) and used for quantitative fluorescent RT-PCR, using an ABI 7900 HT (Applied Biosystems) [8].

Bisulphite sequencing of the $52 \mathrm{CpG}$ sites of the $F M R 1$ promoter region was performed as previously described [8]. A total of 9 clones were sequenced from WT, E3 and MP cell lines before and after treatment with AFQ056.

\section{Results}

The AFQ056 treatment was performed on WT and three FXS lymphoblastoid cell lines. No FMR1-mRNA increase was observed after treatment with AFQ056 in any of the four cell lines, with respect to the untreated controls. All results are summarized in Figure 1. The WT cell line was used to establish the basal level of $F M R 1$ gene transcription. The partial decrease in FMR1 transcription observed in WT at $1 \mathrm{mM}$ AFQ056 after 3 and 8 days of treatment (panel A) was due at least in part to cell mortality. Actually at 8 days post-treatment the cell viability had decreased from 20 to $12 \times 10^{6}$ cells. A second reason is the physiological fluctuations in FMR1 gene transcription previously observed [9]. All cell lines were also treated with $[1 \mu \mathrm{M}] 5$-azadC for 3 and 8 days, respectively, to test the efficiency of transcriptional reactivation (positive control for FMR1 gene reactivation). The 5-azadC showed a mean FMR1 reactivation of $10 \%$ after 3 days and of $25 \%$ after 8 days for E3 and S1 cell lines, while in the WT cell line no significant increase was observed, in accordance with previous results [8]. The MP (size and methylation mosaic) cell line displayed higher levels of FMR1 reactivation $(20 \%$ and 32\% after 3 and 8 days, respectively) according with the residual transcription of the gene in these cells. This latter cell line was derived from an FXS boy and did not show any residual translation on Western blot (data not shown).

In spite of the lack of effect of AFQ056 on FMR1 transcript, we went on to analyze DNA methylation of the 52 cytosines constituting the CpG island of the FMR1 promoter region. Bisulphite sequencing was performed on 9 clones (cells) of untreated WT and on 4 clones each of WT after 3 and 8 days of treatment with $1 \mathrm{mM}$ AFQ056. A total of 9 clones were sequenced for FXS lines E3 and MP and 9 clones for the same FXS cell lines after treatment. As expected, the promoter of the untreated and treated WT cells was entirely unmethylated, while in the untreated FXS cell lines it was fully methylated, with the exception of few clones completely unmethylated in the MP mosaic cell line. AFQ056 treatment had no effect on methylation, leaving the promoter as methylated as in the untreated controls both WT and FXS (Figure 2). 


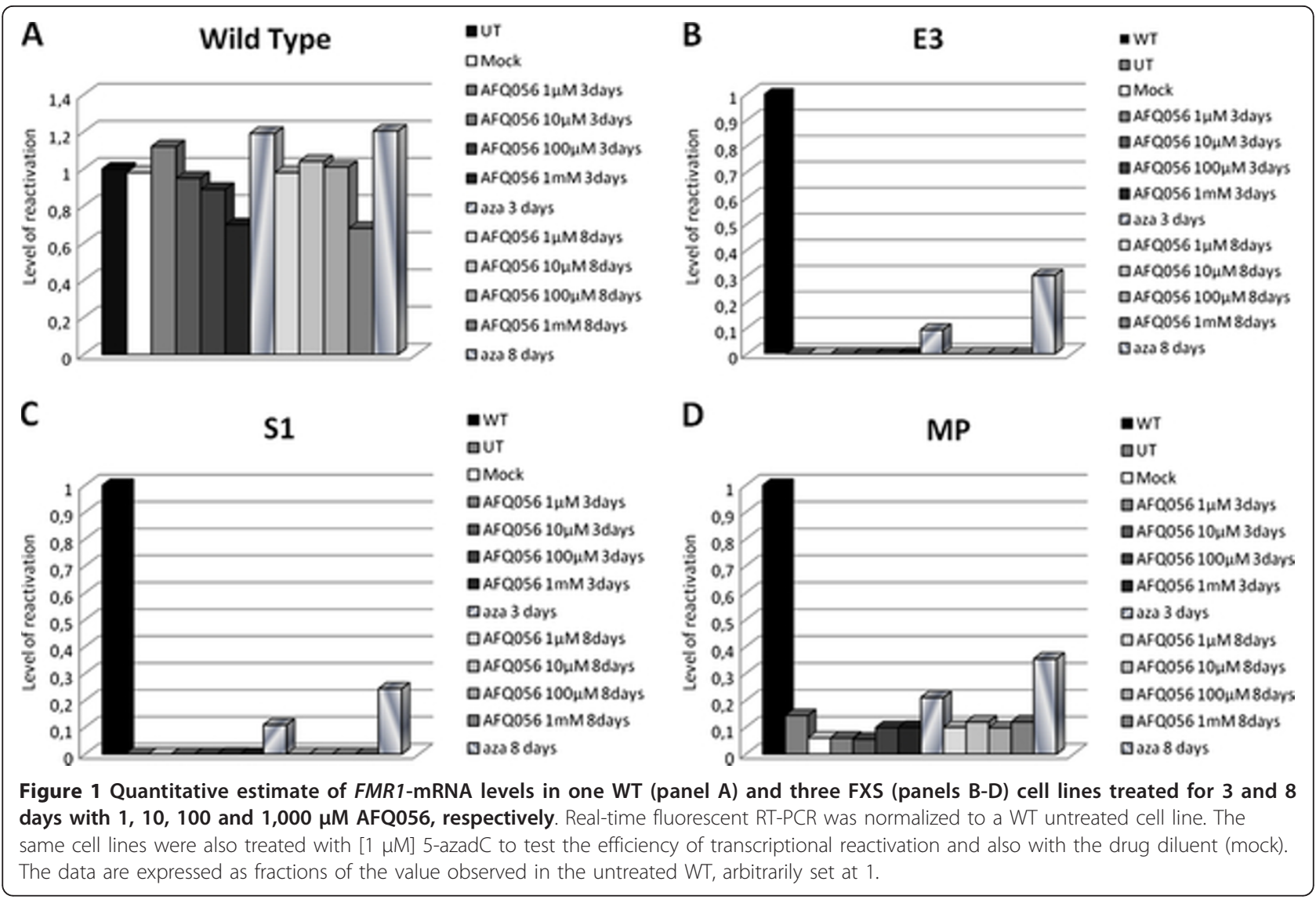

\section{Discussion}

A link between the neurological and behavioural FXS phenotype, due to absence of FMRP, and the upregulation of mGluR5-mediated activities at dendritic synapses was proposed by the so-called mGluR theory [3]. Both FXS patients and Fmrl KO mice show elongated dendritic spines and enhanced mGluR5-mediated long term depression (LTD) due to a perturbation of the mGluR5 signalling [4].

The observations led to consider the possible use of mGluR5 antagonists to treat FXS symptoms. One of the first compounds to be tested was MPEP, as already discussed [5]. A similar effect was obtained with fenobam, another mGluR5 inhibitor [10]. Fenobam was originally used as an anxiolytic agent, even though at that time its molecular target in the brain was not known. Later on, it was discovered that fenobam is a potent and selective negative allosteric modulator of mGluR5 like MPEP, but with a different chemical structure [11]. A small openlabel, single-dose trial of fenobam was conducted to provide an initial evaluation of safety and pharmacokinetics in FXS adults, without any significant adverse effects and with a few beneficial clinical effects [12]. More recently encouraging results were obtained by treating Fmr1 KO mice with AFQ056, a subtype- selective inhibitor of mGluR5, capable of rescuing the prepulse inhibition deficit, as well as the dendritic spine phenotype [6]. This compound had previously been used in a clinical trial of Parkinson disease patients with levodopa-induced dyskinesia, demonstrating antidyskinetic effect without changing the antiparkinsonian effects of dopaminergic therapy [13]. Based on evidence of safety and potential clinical efficacy, AFQ056 was used in a randomized, double-blind, two-treatment, two-period, crossover trial of 30 FXS patients aged 1835 years [7]. In the primary outcome measure (ABC-C score) of this study no statistically significant differences were observed among the treated and untreated groups. However, a secondary exploratory analysis suggested that the response to AFQ056 may be predicted by the methylation status of the FMR1 promoter: subjects with a fully methylated $F M R 1$ promoter showed statistically significant improvement in their behaviour, while carriers of a partially methylated promoter did not. These findings demonstrated that AFQ056 may alleviate stereotypic behaviour, hyperactivity, inappropriate speech and restricted interests and also improve autistic behaviour in FXS subjects whose FMR1 promoter is fully methylated. Our present in vitro study of AFQ056 on three FXS lymphoblastoid cell lines with different 


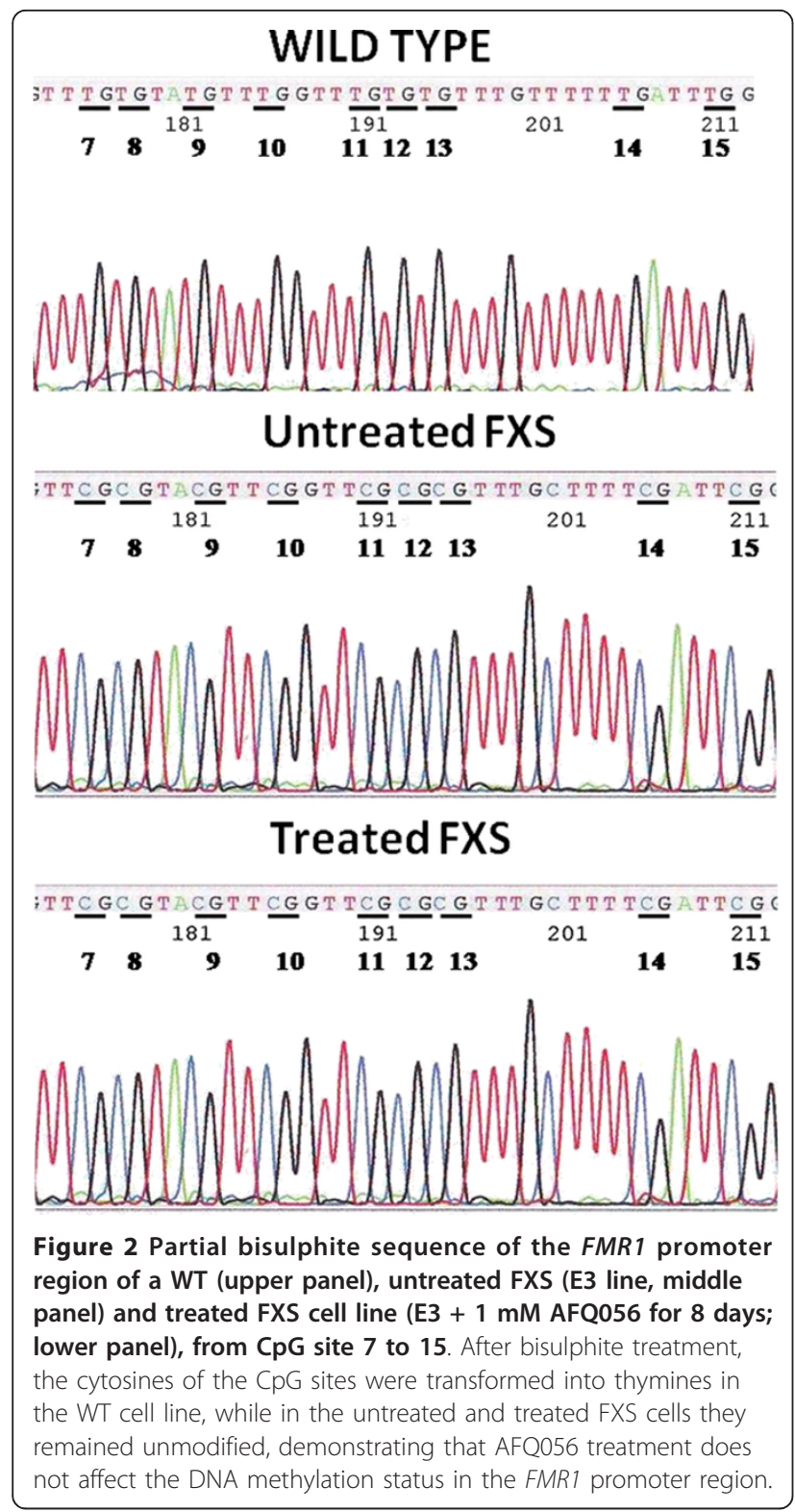

degree of FMR1 promoter methylation now demonstrates that the phenotypic improvement observed in the fully methylated patients is not due to an effect on methylation induced by this drug on the promoter region. Treatment of FXS cell lines with AFQ056 did not cause either an increase in the FMR1 transcription or demethylation of the FMR1 promoter. These findings were consistently reproducible in three different FXS cell lines. The partial decreased transcription observed in WT cell treated with $1 \mathrm{mM}$ of AFQ056 was probably not due to an effect on DNA methylation, but rather to physiological variations in gene transcription and to cell mortality, as indicated by cell count. Clearly, we are aware that results obtained in lymphoblastoid cells may not necessarily reflect the status of neuronal cells, whose behaviour is central to the pathophysiology of FXS.

\section{Conclusion}

Given the results obtained in this study, one could speculate that full methylation of the FMR1 promoter may reflect the activity, or lack thereof, of other proteins interacting with the mGluR5 signalling pathway, thus favouring the beneficial effect of AFQ056 in this subpopulation of FXS patients. An alternative explanation could be that only in the fully methylated patients the mGluR5-mediated signalling is sufficiently high to make the effect of AFQ056 clinically detectable, while this would not be the case in partially methylated patients where the mGluR5 signalling is not so high, due to residual presence of the FMR1 protein.

\section{Acknowledgements}

This work was supported by TELETHON grant GGP10150 and by a FRAXA Foundation grant to G.N.

\section{Author details}

${ }^{1}$ Istituto di Genetica Medica, Università Cattolica del S. Cuore, Rome, Italy. ${ }^{2}$ Novartis Institutes for BioMedical Research, Basel, Switzerland. ${ }^{3}$ Istituto di Genetica Medica, Università Cattolica del Sacro Cuore, Largo F. Vito 1, 00168 Rome, Italy.

\section{Authors' contributions}

The original hypothesis regarding the study was conceived by GN. Compound AFQ056 was provided by BGM and FG. Planning of experiments and study design was performed by ET and approved by all authors.

Sequencing analysis was performed by FP. The draft was written by GN and ET, all authors revised the manuscript and contributed to the discussion. All authors read and approved the final manuscript.

\section{Competing interests}

The authors declare that they have no competing interests.

Received: 29 September 2011 Accepted: 7 March 2012

Published: 7 March 2012

\section{References}

1. Verkerk AJ, Pieretti M, Sutcliffe JS, Fu YH, Kuhl DP, Pizzuti A, et al: Identification of a gene (FMR-1) containing a CGG repeat coincident with a breakpoint cluster region exhibiting length variation in fragile $X$ syndrome. Cell 1991, 65:905-914.

2. Zalfa F, Giorgi M, Primerano B, Moro A, Di Penta A, Reis S, et al: The fragile $X$ syndrome protein FMRP associates with $B C 1$ RNA and regulates the translation of specific mRNAs at synapses. Cell 2003, 112:317-327.

3. Bear M, Huber KM, Warren ST: The mGluR theory of fragile $\mathrm{X}$ mental retardation. Trend Neurosci 2004, 27:370-377.

4. Dölen G, Osterweil E, Rao BS, Smith GB, Auerbach BD, Chattarji S, Bear MF: Correction of fragile X syndrome in mice. Neuron 2007, 56:955-962.

5. Yan QJ, Rammal M, Tranfaglia M, Bauchwitz RP: Suppression of two major fragile $\mathrm{X}$ syndrome mouse model phenotypes by the mGluR5 antagonist MPEP. Neuropharmacology 2005, 49:1053-1066.

6. Levenga J, Hayashi S, de Vrij FM, Koekkoek SK, van der Linde HC, Nieuwenhuizen I, et al: AFQ056, a new mGluR5 antagonist for treatment of fragile $X$ syndrome. Neurobiol Dis 2011, 42:311-317.

7. Jacquemont $\mathrm{S}$, Curie A, des Portes V, Torrioli MG, Berry-Kravis E, Hagerman RJ, et al: Epigenetic modification of the FMR1 gene in fragile $\mathrm{X}$ syndrome is associated with differential response to the mGluR5 antagonist AFQ056. Sci Transl Med 2011, 3:1-9. 
8. Pietrobono R, Pomponi MG, Tabolacci E, Oostra B, Chiurazzi P, Neri G: Quantitative analysis of DNA demethylation and transcriptional reactivation of the FMR gene in fragile X cells treated with 5azadeoxycytidine. Nucleic Acids Res 2002, 30:3278-3285.

9. Tabolacci E, Moscato U, Zalfa F, Bagni C, Chiurazzi P, Neri G: Epigenetic analysis reveals a euchromatic configuration in the FMR unmethylated full mutations. Eur J Hum Genet 2008, 16:1487-1498.

10. de Vrij FM, Levenga J, van der Linde HC, Koekkoek SK, De Zeeuw Cl, Nelson DL, et al: Rescue of behavioral phenotype and neuronal protrusion morphology in Fmr KO mice. Neurobiol Dis 2008, 31:127-132.

11. Porter RH, Jaeschke G, Spooren W, Ballard TM, Büttelmann B, Kolczewski S, et al: Fenobam: a clinically validated nonbenzodiazepine anxiolytic is a potent, selective, and noncompetitive mGlu5 receptor antagonist with inverse agonist activity. J Pharmacol Exp Ther 2005, 315:711-721.

12. Berry-Kravis E, Hessl D, Coffey S, Hervey C, Schneider A, Yuhas J, et al: A pilot open label, single dose trial of fenobam in adults with fragile $X$ syndrome. J Med Genet 2009, 46:266-271.

13. Berg D, Godau J, Trenkwalder C, Eggert K, Csoti I, Storch A, et al: AFQ056 treatment of levodopa-induced dyskinesias: results of 2 randomized controlled trials. Mov Disord 2011, 26:1243-1250.

\section{Pre-publication history}

The pre-publication history for this paper can be accessed here: http://www.biomedcentral.com/1471-2350/13/13/prepub

doi:10.1186/1471-2350-13-13

Cite this article as: Tabolacci et al:: The mGluR5 antagonist AFQ056 does not affect methylation and transcription of the mutant FMR1 gene in vitro. BMC Medical Genetics 2012 13:13.

\section{Submit your next manuscript to BioMed Central and take full advantage of:}

- Convenient online submission

- Thorough peer review

- No space constraints or color figure charges

- Immediate publication on acceptance

- Inclusion in PubMed, CAS, Scopus and Google Scholar

- Research which is freely available for redistribution

Submit your manuscript at www.biomedcentral.com/submit 